\title{
DETERMINATION OF THE STRESS-STRAIN STATE BY STAMPING EXTRUSION OF AXISYMMETRIC PARTS
}

\author{
Ismailov Khasan ${ }^{1}$, Abdullaev Fatkhullaํ, Akhmadaliev Shahkrukh1, Zagidulin Rafail \\ ${ }^{1}$ Tashkent State Technical University \\ Email: red engineer94@mail.ru
}

\begin{abstract}
The article says that in order to solve problems in the field of metal forming by pressure, programs are needed that could take into account all factors in plastic deformation. The article discusses a method for recovering a deformed mesh from a fibrous macrostructure, determines deforming forces by the upper estimate method, and also proposes a method for solving problems of stamping by extrusion using the upper estimate method. Theoretically restored the second family of lines after cutting the workpiece. The pictures of the fibrous macrostructure, the original square cell of the dividing grid, the calibration graph, the diagrams of the main and average stresses, the diagram of the stamp when extruding axisymmetric parts are shown.
\end{abstract}

Keywords: Deformation, Extrusion, Calibration Schedule, Fibrous Structure.

\section{Introduction}

In mechanical engineering, when processing rolled products, a large amount of metal turns into chips. Chip processing requires large capital investments. Therefore, one of the main directions of improving the technology of forging and pressing production is the development and implementation of technological processes of metal forming, which reduce irrational metal losses.

Methods of the theory of plastic deformations based on the phenomenological approach are increasingly being used to design new and improve existing technological processes.

Currently, methods of mathematical modeling of the nature of the metal flow in the stamping processes have been developed, which allow us to solve the problem in a complete formulation. However, to obtain such comprehensive information about the technological process and the quality of the product, modern programs are necessary.

In shop conditions, such software is required that would allow obtaining sufficiently reliable results when determining the energy-force parameters of the process, its stress-strain state, estimating the maximum shape change in one transition without destruction, etc.

\section{Methods}

The existing methods of calculation, including engineering, related to the integration of approximate differential equations together with the plasticity condition, are still quite time-consuming and are not always clear to the process engineer.
Therefore, until now, technologists use well-known analytical formulas obtained for special cases, and, consequently, the calculation results cannot always be used reliably.

In connection with this, the problem has arisen to develop a method for analyzing the technological process of stamping, which would be accessible to a process engineer in shop conditions and would be understandable.

Such a method can be an upper estimate of the deforming force, which is based on the use of a theorem, the essence of which, in turn, is that the power developed by external forces at given speeds is always less than or equal to the power developed by internal forces at kinematically possible speeds.

Among the progressive technological processes are those that allow us to produce high-quality products with minimal labour costs, energy consumption with the application of less deforming forces and with high productivity.

The technology of stamping by extrusion is considered to be among the progressive ones. The advantages of extrusion stamping in comparison with other technological processes are as follows:

- the possibility of manufacturing forgings in shape and size approaching machine-building parts, which allows to minimize the subsequent processing by cutting;

- the high dimensional accuracy (10th-12th quality in the transverse direction) and the surface quality (roughness) of forgings in some cases makes it possible to completely eliminate subsequent machining;

- the hardening that occurs as a result of cold deformation makes it possible to replace low-alloy 
and medium-carbon steels with low-carbon ones; - the metal using coefficient can be increased to 0.98 $\%$;

- the possibility of mechanization and automation will improve working conditions;

- high productivity with low labor costs;

- the possibility of reducing the number of transitions and operations compared to cold stamping from sheet material.

Extrusion stamping is increasingly used in agricultural machinery, automotive and other industries.

The limiting factor of the propagation of stamping by extrusion is the need to create high specific forces that in some cases exceed 4-6 times the flow stress of the processed material.

Methods for calculating the forces necessary for deforming the workpiece under the conditions of stamping by extrusion do not always allow you to quickly perform the calculation in shop conditions.

In the presented article, a method for solving problems of stamping by extrusion using the upper bound method is proposed.

As a result, prerequisites are created for the use of extrusion stamping in the production of rod parts of various configurations with a relatively high coefficient of metal using.

As a method for determining the parameters of the kinematic deformed and stressed state, an experimental and analytical method of coordinate grids reconstructed from a fibrous macrostructure was chosen. The determination of the deformed and stressed states was carried out on the basis of the theory of plastic flow, which implies observing the cells of the coordinate grid at any moment of shape change.

In the study of axisymmetric deformation by the method of a wavy macrostructure, one type of samples can be used to restore a distorted dividing grid - with the direction of the original macrostructure along the axis of symmetry. When cutting such a sample along the meridional plane and subsequent etching, we will have only one family of lines of the distorted grid associated with the fibers of the macrostructure. Let's restore the second family of coordinate grid lines. To do this, imagine that this family of horizontal lines was plotted on a plane passing through the axis of symmetry of the sample. Moreover, we will examine the grid with the original square cell.

To restore the second-a family of lines from the labels located on the side surface, we use the incompressible conditions in the following sense. When the sample is deformed, the shape of the grid cell and its area will change. The volume of the body formed by the rotation of this cell around the axis of symmetry will remain constant during the entire shape change.
The volume of the body formed by the rotation of the original square cell with an area of $S_{\varepsilon q}=\delta^{2}$, is easily calculated:

$$
V_{s}=2 \pi \cdot R_{e g} \cdot S_{s q}
$$

where $R_{\text {eg. }}$ - the radius of rotation of the center of gravity of the section of the original square cell around the axis of symmetry (Fig. 1).

$$
R_{e g .}=2\left(j-n_{0}+0.5\right) \cdot \delta^{\prime}
$$

$n_{0}$ - the ordinal number of the vertical line that coincides with the axis of symmetry.

In our case $n_{0}=4$, since we need to extend the results of the deformed state to three auxiliary rows of points located outside the focus of plastic deformations. This is due to the process of smoothing the experimental fields of deformations and stresses.

After the deformation, the cell turned from a square into a quadrilateral of any shape, but the volume remained constant. This is the basis for the construction of a horizontal family of lines.

The quadrilateral $(1,2,3,4)$ can be divided into two triangles $(2,3,4)$ and $(1,2,4)$. Then the volume $\mathrm{V}$ will consist of the volumes obtained by rotating the triangles around the axis of symmetry.

$$
\mathrm{V}=\mathrm{V}_{1}+\mathrm{V}_{2}
$$

$\mathrm{V}_{1}$ and $\mathrm{V}_{2}$ are calculated according to the rules of Gulden: when a flat figure rotates relative to an axis lying in the plane of this figure and does not intersect it, a body is formed whose volume is equal to the product of the area of the figure by the circumference described by the center of gravity, i.e.

$$
\begin{aligned}
& V_{1}=2 \pi \cdot R_{c \cdot g 1} \cdot S_{1} \\
& V_{2}=2 \pi \cdot R_{c, g 2} \cdot S_{2}
\end{aligned}
$$

where $R_{-}$(c. t1), R_(c. t2) are the centres of gravity of the triangles, respectively, $(2,3,4)$ and $(1,2,4)$;

$$
\begin{aligned}
& S_{1}=S_{2}-\text { are the areas of the same triangles. } \\
& S_{1}=\frac{1}{2}\left(\rho_{2} z_{3}-\rho_{3} z_{2}+\rho_{3} z_{4}-\rho_{4} z_{3}+\rho_{4} z_{2}-\rho_{2} z_{4}\right) \\
& S_{2}=\frac{1}{2}\left(\rho_{1} z_{2}-\rho_{2} z_{1}+\rho_{2} z_{4}-\rho_{4} z_{2}+\rho_{4} z_{1}-\rho_{1} z_{4}\right),
\end{aligned}
$$

In formulas 2.5, $\rho_{i}$ and $z_{\bar{i}}$ - are the coordinates of $\rho$ and $\mathrm{z}$ of the corresponding points $1,2,3,4$.

$$
\begin{aligned}
& R_{c \cdot g 1}=\frac{1}{3}\left(\rho_{2}+\rho_{3}+\rho_{1}\right) \\
& R_{c \cdot g 2}=\frac{1}{3}\left(\rho_{1}+\rho_{2}+\rho_{4}\right)
\end{aligned}
$$


According to the incompressibility conditions, there should be

$$
V_{s}=V_{1}+V_{2}
$$

Starting to consider the cells from the lower boundary of the focus of plastic deformation, where the initial data of the vertical and horizontal lines are not distorted, we can determine the values of the coordinates $\rho$ and z 1-ой и 2-ой points of the cell. From a series of cells lying on an undistorted horizontal line, we will select the cell that is in contact with the axis of symmetry. This will allow us to calculate the coordinates $\rho$ and $\mathrm{z}$ of point 4 from the condition of equality of the volumes of the bodies formed by the rotation of the original and distorted triangle $(1,2,4)$ around the $\mathrm{z}$ axis.

So, from equation (2.7), the coordinates of only one point remain unknown - point 3 , which must be found by a sequential approximation, present in equation (2.3) pairs of values $\rho_{3}$ and $z_{3}$, that satisfy the equation describing the shape of the fibre of the first family on which point 3 should lie, checking the fulfilment of condition (2.7), if equation (2.7) is fulfilled, then a pair of coordinate values $\rho_{3}$ and $z_{3}$ will be the desired one.

Moving from one cell to another from bottom to top and from left to right and repeating the specified procedure, we find the coordinates of all points of intersection of lines of both families.

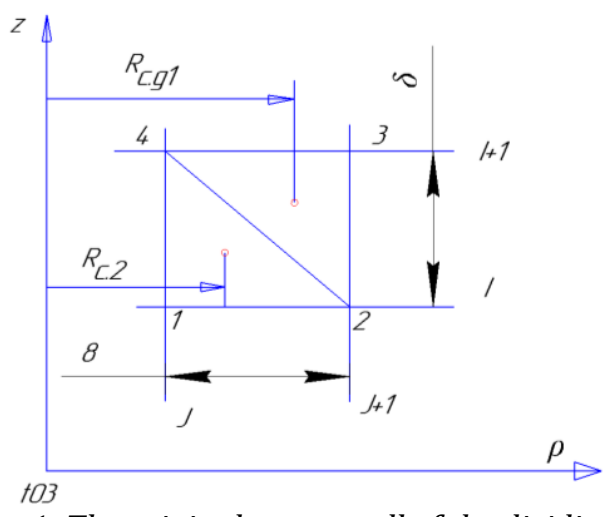

Figure 1: The original square cell of the dividing grid

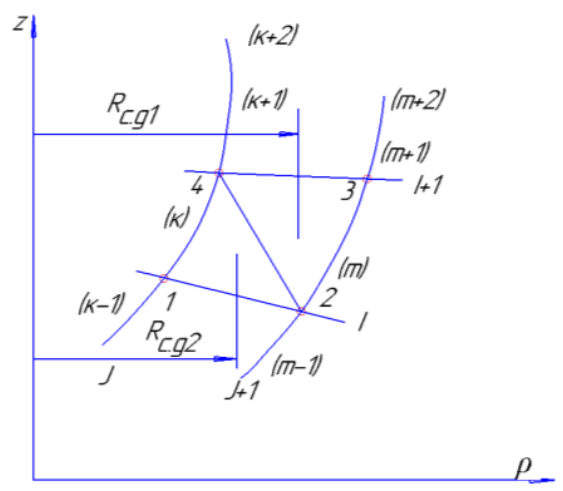

Figure 2: Scheme for calculating the coordinates of the nodes of the dividing grid
The stress-strain state was also studied by combining the hardness method and determining deformations from a fibrous macrostructure. For the research, blanks from $\mathrm{AD}-1$ with a pronounced macrostructure were used. The blanks were stamped with various filling of the flange cavity, thickening. The samples were then cut into planes parallel to the meridional section. The resulting surface was sanded and polished. A grid with a step of $1 \mathrm{~mm}$ was applied on this surface by scratching with a chisel using a special device on a microscope.

After that, the Vickers hardness was measured in the nodes of the square grid of the deformed workpiece. Measurements were carried out on cells arranged in a staggered order to exclude the influence of deformations of the neighboring cell. 150-200 hardness measurements were performed on each sample under a load of $5 \mathrm{~kg}$. Such a load provided a clear imprint and a sufficient number of them in the deformed area.

The calibration graph Fig. 3, linking the value of the Vickers hardness HV with the stress intensity $\sigma_{i}$ and the deformation $\varepsilon_{\mathrm{i}}$, was constructed based on the results of compression tests. For this purpose, the samples from $\mathrm{AD}-1$ were deformed with various degrees of deformation under conditions when contact friction is almost absent. 30 samples were deformed. Grinding and polishing was carried out on the surface along the generatrix and at 5-6 of its points, sufficiently close to the center of the sample section. The Vickers hardness HV was measured. The corresponding intensity of stresses and deformations was determined by the relations:

$$
G_{i}=\frac{4 P}{\pi d^{2}} ; \varepsilon_{i}=\ln \frac{h_{H}}{h_{K}}
$$

where P - compressive force; $\mathrm{d}$ - diameter of the workpiece; $h_{H} h_{K}$ - sample height before and after draft.

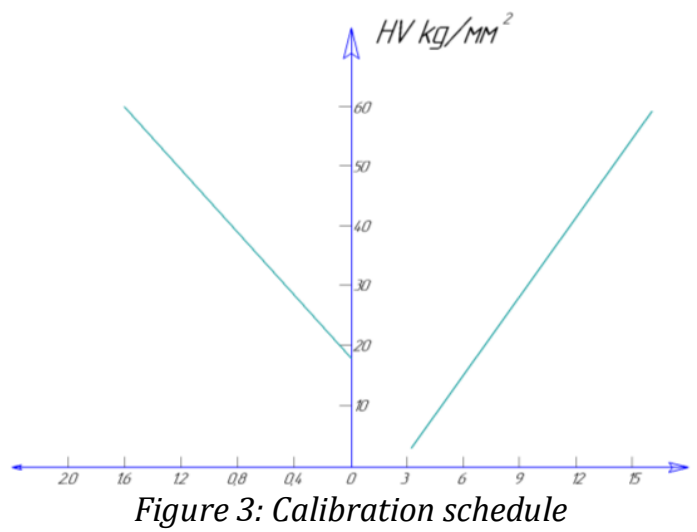

According to the measured hardness field on the deformed samples, the stress intensity was determined using the obtained calibration graph.

After measuring the hardness, the surfaces where the measurements were made were again sanded 
and polished. The surface prepared in this way to reveal the fibrous macrostructure was etched in a mixture of acids: one volume of concentrated hydrochloric acid, one volume of concentrated hydrofluoric acid and one volume of concentrated nitric acid. Then the surface was thoroughly washed in hot running water and dried. Figure 4 shows photos of the fibrous macrostructure of the studied samples.

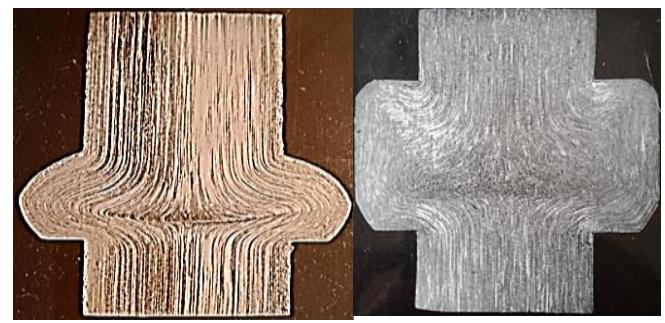

Figure 4: Fibrous structure of the sample

When solving technological problems on the basis of the upper bound theorem, it is necessary to develop a method for calculating the energy-force parameters of deformation of the workpiece.

To choose forging and stamping equipment in the conditions of automated production, it is necessary to know the power of the flywheel electric drive of the press and the maximum deforming force already at the design stage of the technological process.

To determine the power of the flywheel electric drive, it is necessary to calculate the deformation work spent on deforming the workpiece during a technological operation or transition. To do this, it is necessary to build the expected graph of the deforming force along the press slider.

According to the upper bound theorem: "The power of real external forces at given speeds is always less than or equal to the power of external and internal forces at kinematically possible", i.e.

$\int_{F_{V}} q V_{0} d F \leq \sigma_{s} \int_{V} \xi_{i}^{*} d V+\frac{\sigma_{s}}{\sqrt{3}} \int_{f}\left(V_{1}^{*}+V_{2}^{*}\right) d f-\mu_{s} \sigma_{s} \int_{F_{V}} V^{*} d F$

where $q$ - is the intensity of the actual external forces on the part of the surface of the workpiece $F_{V}$, on which the flow velocities $V_{0}$ are given;

$\xi_{i}^{*}$ - the intensity of kinematically possible strain rates;

$V_{1}^{*}+V_{2}^{*}$ - kinematically possible flow velocities on shear velocity discontinuity surfaces;

$V^{*}$ - the kinematically possible flow velocity on a part of the surface of the workpiece $F_{p}$, on which external forces are specified (in technological problems of pressure treatment, such forces are friction forces);

$\sigma_{s}-$ yield stress (usually set according to the diagram of an ideal rigid-plastic body);;

$\mu_{g}-$ the friction factor.
Equation 3 can be solved if the kinematically possible flow velocities are known. The kinetically possible velocities are understood as flow velocities that satisfy the boundary conditions and the condition of volume constancy.

To satisfy the condition of volume constancy, it is necessary to choose one component of the flow velocity arbitrarily, satisfying only the boundary conditions, and the second-from the solution of the differential equation expressing the condition of volume constancy. After selecting the kinematically possible flow velocities, it is necessary to determine the kinematically possible deformation velocities and their intensity according to the known dependencies.

After completing the above tasks, it is necessary to determine the shape and size of the deformation center, which play an important role in obtaining parts of the desired shape without destruction, as well as to design die tooling for stamping by extrusion on double-acting presses.

As a result of the conducted studies, it was found that such factors as the height of the extruded element and the diameter of the inscribed circle have the greatest influence on the formation of the deformation focus during the extrusion process.

Experiments have shown that the central part of the forging undergoes the most intense deformation during the extrusion process. When one-way metal is fed into the thickening cavity, the maximum values of deformations are located at the lower hub, which is explained by the fact that the metal entering the cavity from the upper hub shifts the metal to the lower boundary of the deformation center, where shear deformations accumulate due to the movement of the metal in a plastic state relative to the rigid boundary of the hub. From the side of the upper boundary of the deformation through the upper boundary of the deformation center, undeformed metal continuously enters it. The peripheral layers of the metal are slightly deformed in the radial direction until the side wall of the container touches. At this moment, the circumferential deformations reach a maximum on the outer side surface of the flange. Subsequent filling of the flange cavity with metal by changing the signs of deformation in the radial and the shape of the boundaries of the zone of intense deformation located under the punch at the free flow stage is determined by the ratio of the dimensions of the cavity height to the diameter of the receiving container. At the ratio $\mathrm{H}_{\mathrm{f}} / \mathrm{D}_{0}>0.27$ the focus of deformation has a biconcave lens. At ratios of $0,23 \leq H_{f} / D_{0} \leq 0,27$ the focus takes the form of a cylinder. At ratios of $0,23<\mathrm{H}_{\mathrm{f}} / \mathrm{D}_{0}$ the focus resembles a biconvex shape.

The obtained data confirm the applicability of the computational model with rectilinear boundaries of the focus of plastic deformation. 
An analysis of the nomenclature of forgings at enterprises showed that parts with flanges, thickenings and ledges have become very common. The obtained data confirm the applicability of the computational model with rectilinear boundaries of the deformation focus.

Stress-strain state of the workpiece during radial extrusion.

According to the pre-established relationship between the hardness and intensity of the stress state and the measured hardness in the studied zones of the extruded workpiece, it is possible to judge the degree of past deformation and the intensity of the stress state at the required stage of deformation. Using this method, the isolines HV and $\mathrm{G}_{\mathrm{i}}$ are constructed. It can be seen from the figures that the maximum hardening zone with a one-sided metal supply is adjacent to the lower boundary of the deformation center, and with a two-sided metal supply it moves to the horizontal axis of symmetry.
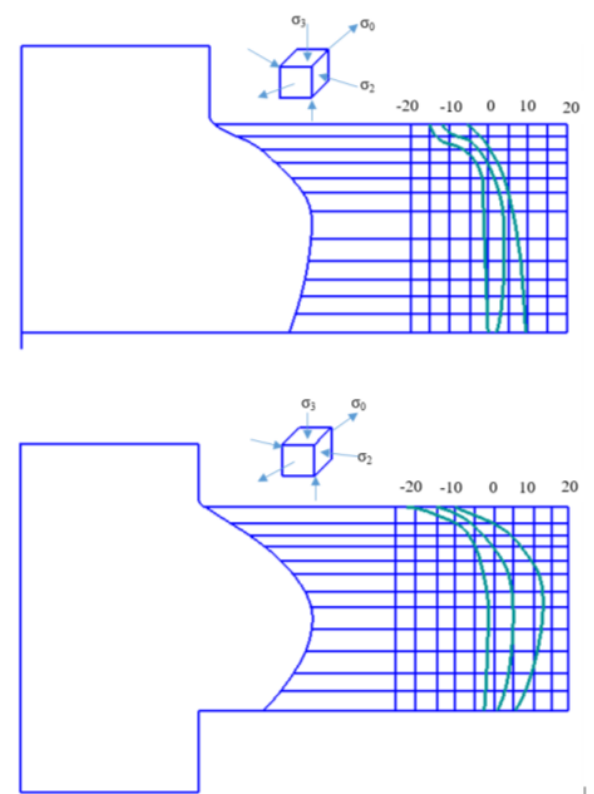

Figure 5: The main stresses on the side contour of the workpiece

The main stresses on the side contour can be calculated from the relations of the deformation theory.

$$
\left\{\begin{array}{l}
\sigma_{\theta}-\sigma=\frac{2}{3} \cdot \frac{\sigma_{i}}{\varepsilon_{i}} \varepsilon_{\theta} \\
\sigma_{2}-\sigma=\frac{2}{3} \cdot \frac{\sigma_{i}}{\varepsilon_{i}} \varepsilon_{2} \\
\sigma_{3}-\sigma=\frac{2}{3} \cdot \frac{\sigma_{i}}{\varepsilon_{i}} \varepsilon_{3}
\end{array}\right.
$$

If the axis 2 is directed to the normal to the side contour, then $\sigma_{2}=0$, as a result of this, the ratio 4 follows

$$
\sigma=-\frac{2}{g} \cdot \frac{\sigma_{i}}{\varepsilon_{i}} \varepsilon_{2},
$$

Substituting the ratio 5 in the ratio 4 we get,

$$
\begin{aligned}
\sigma_{\theta} & =\frac{2}{3} \cdot \frac{\sigma_{\mathrm{i}}}{\varepsilon_{\mathrm{i}}}\left(2 \varepsilon_{\theta}+\varepsilon_{\mathrm{a}}\right) \\
\sigma_{\mathrm{a}} & =\frac{2}{3} \cdot \frac{\sigma_{\mathrm{i}}}{\varepsilon_{\mathrm{i}}}\left(\varepsilon_{\theta}+2 \varepsilon_{\mathrm{a}}\right.
\end{aligned}
$$

The diagrams of the main and average stresses obtained by this method are plotted in Figures 5-6. The circumferential stresses at the equator of the side surface are tensile, and the axial stresses tend to decrease, the stress state is close to linear tension, which leads to a decrease in the plasticity of the deformable metal, therefore, the most dangerous zone from the point of view of the possibility of destruction of the workpiece is the zone on the side surface of the flange.

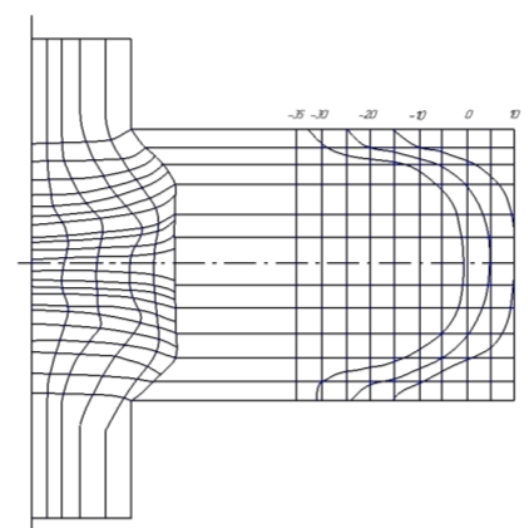

Figure 5: Main stresses on the side contour of the workpiece (double-sided extrusion)

The die tooling is designed taking into account the design features of forging and pressing machines. The stamp consists of support plates I and II, interconnected by guide columns 17 . A punch 5 is attached to the plate of the inner slider 2 , and the upper plate of the stamp 3 with a half - matrix 7 is attached to the outer slider, the lower half-matrix 9 has a movement in the cage 10 to ensure a better blank in the stamp cavity. The forging from the lower half-matrix is pushed out by the press pusher through the intermediate rod 14 . The working tool for radial extrusion on double-acting presses consists of two half - matrices-the upper 7 and lower 9 , a punch 5 , a punch holder 4 and an ejector 18 . The design of the stamp provides a quick replacement of half-matrices. One of the main requirements for the material of half-matrices is resistance to wear. Along with high wear resistance and heat resistance, the half-matrix material should have greater tensile strength and impact strength. As a result of heat treatment, the hardness of the working surface is set in the range HB 45-50 units. Since the punch experiences higher specific loads, it is advisable to process it for a hardness of HB 50-55 units. 


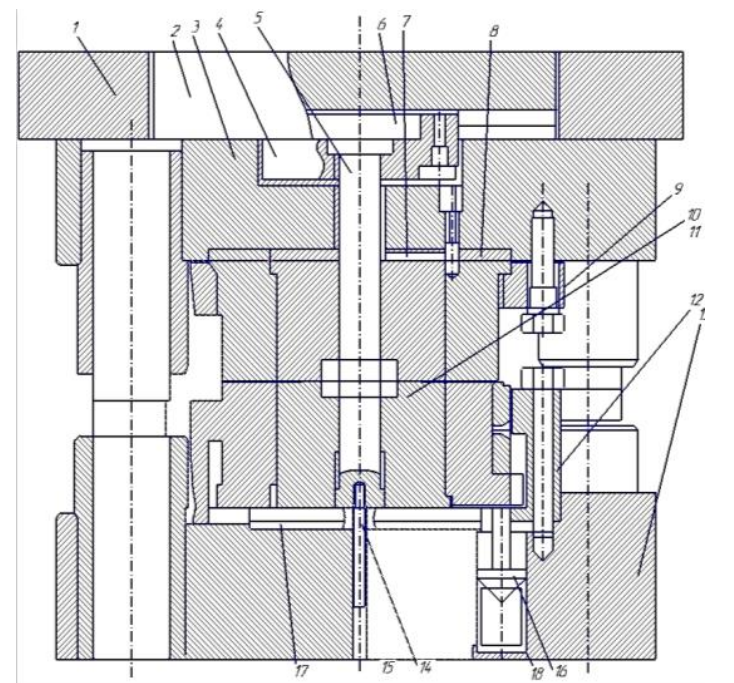

Figure 7: The scheme of the recommended die tooling

The experience of stamping has shown that it is advisable to carry out nitriding of the main elements of the stamp, since this process allows you to get a high hardness of the working surface and does not require subsequent processing.

\section{Conclusions}

As a result of the research, scientifically based technological and technical solutions are given, consisting in the development of a generalized method for calculating the stress-strain state of the workpiece and an upper estimate of the energy-force parameters of the process for an axisymmetric stress state. The developed method is used to calculate the deformed and stressed states in the processes of stamping by extrusion.

\section{References}

[1] A. G. Ovchinnikov, E. P. Unksov; Theory of forging and stamping, Publisher: Mashinostroenie, 1992 y.

[2] P. I. Polukhina; Theoretical foundations of metal forming (theory of plasticity), Moscow, Publisher: Metallurgy, $1980 \mathrm{y}$.

[3] M. V. Storozhev and E. A. Popov; Theory of metal forming, Publisher: Mashinostroenie, 1971.
[4] L. I. Zhivov, A. G. Ovchinnikov, E. N. Skladchikov; Forging and stamping equipment, Moscow, Bauman Moscow State Technical University, 2006.

[5] Umarov, E., Mardonov, U., Abdirakhmonov, K., Eshkulov, A., \& Rakhmatov, B. (2021). Effect of magnetic field on the physical and chemical properties of flowing lubricating cooling liquids used in the manufacturing process. IIUM Engineering Journal, Volume 22, Issue 2, Pp: 327 338, $2021 . \quad$ doi: https://doi.org/10.31436/iiumej.v22i2.1768.

[6] Erkin, U., Umidjon, M., \& Umida, S. (2021, September). Application of Magnetic Field on Lubricating Cooling Technological Condition in Metal Cutting Process. In International Conference on Reliable Systems Engineering (pp. 100-106). Springer, Cham.

[7] E.O. Umarov, U.T. Mardonov, U.K. Shoazimova; Influence of the Magnetic Field on the Viscosity Coefficient of Lubricoolant that is used in the Cutting Proces. International Journal of Mechatronics and Applied Mechanics, Volume 8, Issue 2, Pp: 144-149, 2020.

[8] T. Nodir, T. Sherzod, Z. Ruslan, T. Sarvar, \& B. Azamat. Studying the scientific and technological bases for the processing of dumping copper and aluminum slags. Journal of Critical Reviews, Volume 7, Issue 11, Pp: 441-444, 2020. doi: http://dx.doi.org/10.31838/jcr.07.05.95.

[9] Nodir, T., Sarvar, T., Andrey, J., \& Yahyojon, M.; Mathematical Model for Calculating Heat Exchange. In International Conference on Reliable Systems Engineering, Springer, Cham, Pp: 243-249, 2021.

[10] Turakhodjaev, N., Tursunbaev, S., Tashbulatov, S., \& Kuchkorova, M.; Analysis of technological solutions for reducing the copper concentration in slags from oxygen-flare smelting of copper sulfide concentrates. Journal of Critical Reviews, Volume 7, Issue 5, Pp: 449-452, 2020.

[11] Turakhodjaev, N., Akramov, M., Turakhujaeva, S., Turakhujaeva, A., Kamalov, J.; Calculation of the heat exchange process for geometric parameters International Journal of Mechatronics and Applied Mechanicsthis link is disabled, Volume 1, Issue 9, Pp: 90-95, 2021. 